\title{
Magamp application and limitation for multiwinding flyback converter
}

\author{
C.-C. Wen and C.-L. Chen
}

\begin{abstract}
A new magamp technique for multiwinding flyback converters is proposed. Ideal operating principle and analysis are presented. The practical circuit operation is limited due to the nonideal component characteristics. An analytical model for studying the phenomenon is provided. Based on the model, the mechanism of the boundary condition that causes the converter to be out of regulation is explored. Experimental verifications on a $20 \mathrm{~W}$ two-output flyback converter are conducted. They illustrate the effectiveness of the proposed magamp approach and the accuracy of the presented analytical model.
\end{abstract}

\begin{tabular}{|c|c|}
\hline$C_{C}$ & capacitance of RC clamp snubber \\
\hline$i_{M}$ & instantaneous current of $L_{M}$ \\
\hline$I_{M A}$ & average current of $L_{M}$ \\
\hline$I_{M P i}$ & $\begin{array}{l}\text { peak current of } L_{M} \text { at the end of the } i \text { th } \\
\text { time interval }\end{array}$ \\
\hline$i_{P}, i_{1}, i_{2}$ & $\begin{array}{l}\text { instantaneous current of } L_{K P}, L_{K 1} \text { and } \\
L_{K 2} \text {, respectively }\end{array}$ \\
\hline$I_{P A}, I_{1 A}, I_{2 A}$ & $\begin{array}{l}\text { average currents of } L_{K P}, L_{K 1} \text { and } L_{K 2} \text {, } \\
\text { respectively }\end{array}$ \\
\hline$I_{P P i}, I_{1 P i}, I_{2 P i}$ & $\begin{array}{l}\text { peak currents of } L_{K P}, L_{K 1} \text { and } L_{K 2} \text {, } \\
\text { respectively at the end of the } i \text { th time } \\
\text { interval }\end{array}$ \\
\hline$I_{0}$ & $\begin{array}{l}\text { initial current of } L_{M} \text { at the beginning of } \\
T_{1} \text { time interval }\end{array}$ \\
\hline$K_{P}, K_{1}, K_{2}$ & $\begin{array}{l}\text { inductance factors where } K_{p}=L_{M} / L_{K P}, \\
K_{1}=L_{M} / L_{K 1} \text { and } K_{2}=L_{M} / L_{K 2}+N_{2}^{2} L_{S R}\end{array}$ \\
\hline$L_{M}:$ & magnetising inductance of transformer \\
\hline$L_{S R}$ & $\begin{array}{l}\text { saturated inductance of saturable reactor } \\
S R\end{array}$ \\
\hline$N_{1}, N_{2}$ & $\begin{array}{l}\text { turn ratios of transformer where } N_{1}= \\
n_{p} / n_{1} \text { and } N_{2}=n_{p} / n_{2}\end{array}$ \\
\hline$T$ & switching period \\
\hline$n_{P}, n_{1}, n_{2}$ & $\begin{array}{l}\text { winding turns of transformer for primary } \\
\text { winding, secondary winding } 1 \text { and sec- } \\
\text { ondary winding } 2 \text {, respectively }\end{array}$ \\
\hline$T_{i}$ & $i$ th time interval where $i$ is $1-5$ \\
\hline$V_{C}$ & voltage across $C_{C}$ \\
\hline$V_{g}$ & input DC voltage \\
\hline$R_{C}$ & resistance of RC clamp snubber \\
\hline$V_{M i}$ & $\begin{array}{l}\text { voltage across } L_{M} \text { during the } i \text { th time } \\
\text { interval }\end{array}$ \\
\hline$V_{1}, V_{2}$ & $\begin{array}{lll}\text { output voltage } 1 \text { and } 2 \text { where } \\
V_{1}=N_{1} \cdot V_{O 1}, V_{2}=N_{2} \cdot V_{O 2}\end{array}$ \\
\hline
\end{tabular}

(C) IEE, 2005

IEE Proceedings online no. 20040829

doi:10.1049/ip-epa:20040829

Paper first received 12th January and in revised form 3rd June 2004

The authors are with the Graduate Institute of Electronics Engineering and Department of Electrical Engineering, National Taiwan University, Taipei, Taiwan
$L_{K P}, L_{K 1}, L_{K 2} \quad$ leakage inductances of transformer for primary winding, secondary winding 1 and secondary winding 2 , respectively

\section{Introduction}

Among the variety of switching-mode power converters, the flyback converter is a favourite choice: for design engineers in low power applications. The major merits of the flyback converter are: low part count, effective cost, quick dynamic response and simple multi-output structure. In industrial design of the multi-output flyback converter, a weighted voltage control scheme is often used to maintain regulation for all outputs. However, it does not reduce the total output error by adjusting the weighting factors. It only shifts the error to the other outputs [1]. Another disadvantage is that it is hard to arrange the transformer structure to make the output voltage be in the centre of regulation. Some previous papers [2,3] have improved the cross-regulation of the multi-output flyback converter. However, in some applications, such as the onboard power supply of TFT-LCD monitor, stringent regulation is required to prevent the interference effect on the display panel. A postregulator is added to meet the regulation requirements. Among the different postregulation approaches, the magnetic amplifier (magamp) regulator has been popular for years. Compared with other postregulation schemes, the magamp postregulator is one of the most reliable, efficient and cost-effective solutions.

In recent decades, the magamp approach has mostly been applied in forward-type converters. Research on the use of the magamp for forward converters has also been widely reported, including: resetting methods, design guidelines and limitations [4-6]. There has been little research on the use of the magamp for flyback converters. A magamp technique for the flyback converter is presented in [7]. The main drawback is that the main output for the PWM feedback is restricted to the output with higher voltage, even with light rated output current. In this paper, a new magamp technique for flyback converters with multiple output windings is proposed [8]. The feedback loop and winding turn ratio can be chosen. This feature provides 
more freedom to achieve optimum performance. For a practical design, the operating condition is limited due to the nonideal characteristics of the components.

\section{Basic operation principle}

Figure 1 shows the diagram of the multiwinding flyback converter with a magamp output. For simplicity, a twooutput flyback converter is presented. As we know, in forward converter, the secondary outputs of the main transformer can be viewed as voltage sources and transfer energy to each output simultaneously. However, in the flyback converter, the operating principle is completely different. The flyback transformer can be viewed as a current source. The current time-sharing technique is applied. Figure 2 shows some waveforms in continuous current mode assuming ideal components. The basic operations are described as follows.

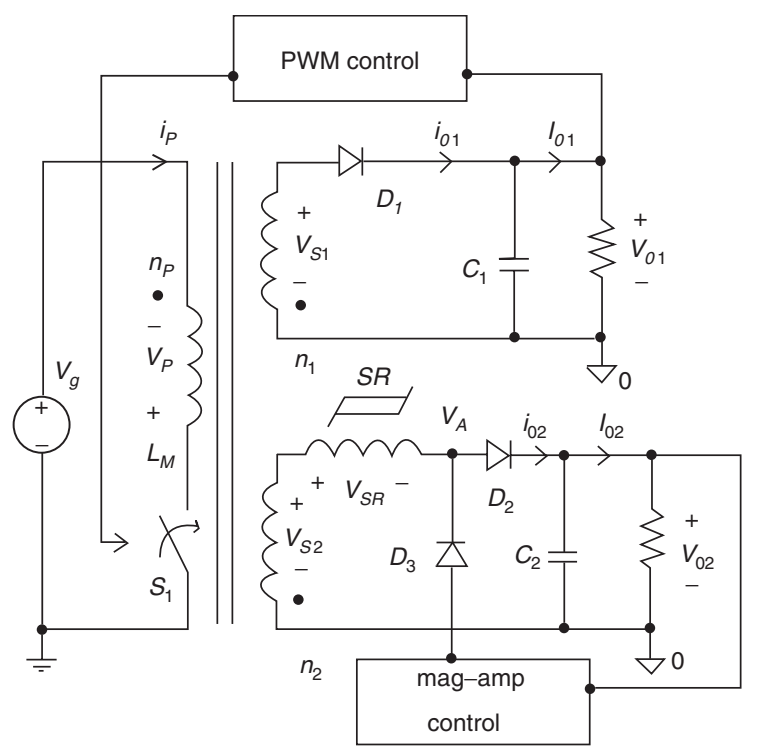

Fig. 1 Diagram of multiwinding flyback converter with a magamp output
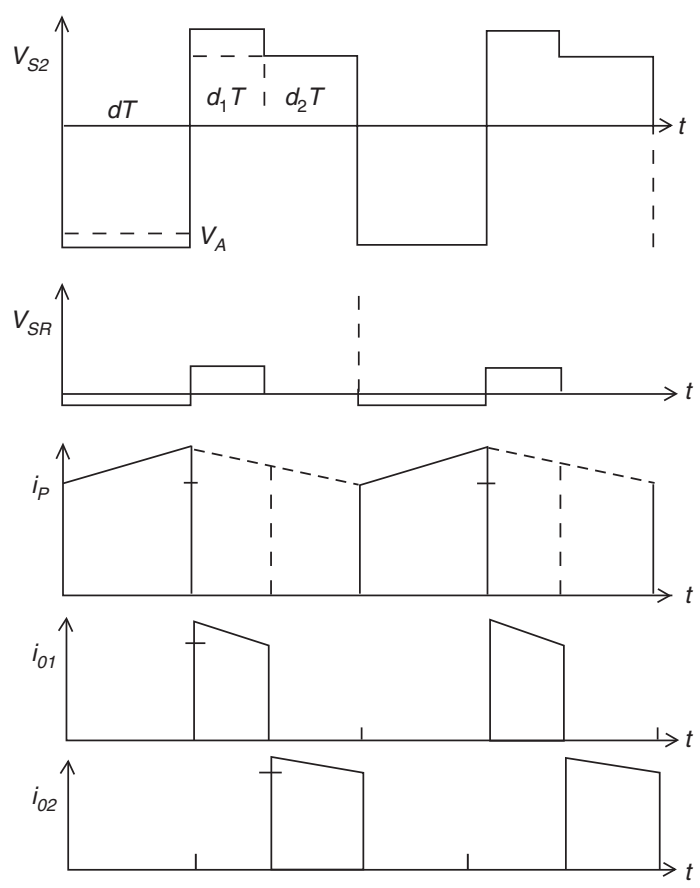

Fig. 2 Some key waveforms in flyback converter with a magamp regulator
In the time period $d T$, the main switch $S_{1}$ is on and the input voltage $V_{g}$ charges the magnetising inductance $L_{M}$, where $d$ is the turn-on duty cycle and $T$ is the switching period. In the flyback converter, the main transformer is not only a common transformer but also an inductor to supply the energy to the outputs. At this period, the saturable reactor $S R$ is reset by the voltage difference between the reverse secondary winding voltage $V_{S 2}$ and the magamp controlled voltage $V_{A}$.

During the time period $d_{1} T$, the switch is turned off and the energy in the magnetising inductance is released to the output $V_{O 1}$. In this period, the diode $D_{1}$ is on and the secondary winding voltage $V_{S 1}$ is clamped at the output voltage $V_{O 1}$. The saturable reactor $S R$ is in the blocking state and is set with the voltage difference between the secondary winding voltage $V_{S 2}$ and the output voltage $V_{O 2}$. Ideally the stored energy in the main transformer is only supplied to the output $V_{O 1}$.

As soon as the saturable reactor $S R$ is saturated, the diode $D_{2}$ comes on and the secondary winding $V_{S 2}$ is clamped at the output voltage $V_{O 2}$. Since the reflected voltage on the winding voltage $V_{S 1}$ is lower than the output voltage $V_{O 1}$, the diode $D_{1}$ becomes reverse bias and is turned off. As a result, ideally the stored energy of the main transformer is only transferred to the output $V_{O 2}$ during the time period $d_{2} T$. At the end of the switching period, the main switch is turned on again and the saturable reactor $S R$ is reset ready for next switching period.

According to the above descriptions, the following equation is needed to be satisfied to ensure the magamp set operation:

$$
\frac{V_{O 1}}{n_{1}}>\frac{V_{O 2}}{n_{2}}
$$

For the volt-sec and balance rule of the main transformer, we may have

$$
\begin{gathered}
V_{g} \cdot d=\frac{n_{P}}{n_{1}} \cdot V_{O 1} \cdot d_{1}+\frac{n_{P}}{n_{2}} \cdot V_{O 2} \cdot d_{2} \\
d+d_{1}+d_{2}=1
\end{gathered}
$$

where $n_{P}, n_{1}$ and $n_{2}$ are winding turns for primary winding, output winding 1 and output winding 2 , respectively.

To achieve continuity of magnetising current when the current flowing through output $V_{O 1}$ is switched to $V_{O 2}$, the following equation is satisfied:

$$
\frac{I_{O 1} \cdot \frac{n_{1}}{n_{P}}}{d_{1}}=\frac{I_{O 2} \cdot \frac{n_{2}}{n_{P}}}{d_{2}}+\frac{1}{2} \cdot \frac{V_{g} \cdot d \cdot T}{L_{M}}
$$

where $I_{O 1}$ and $I_{O 2}$ are the average current of output 1 and output 2 , respectively.

Substituting (2) and (3) into (4) yields

$$
d^{3}+p \cdot d^{2}+q \cdot d+r=0
$$

where $p=-\left(\frac{V_{1}}{V_{g}+V_{1}}+\frac{V_{2}}{V_{g}+V_{2}}\right)$

$$
\begin{aligned}
q & =\frac{2 \cdot L_{M}}{T} \cdot \frac{\left(V_{2}-V_{1}\right)}{V_{g}} \cdot\left[\frac{I_{1}}{V_{g}+V_{2}}+\frac{I_{2}}{V_{g}+V_{1}}\right] \\
& +\frac{V_{1} \cdot V_{2}}{\left(V_{g}+V_{1}\right)\left(V_{g}+V_{2}\right)} \\
r & =-\frac{2 \cdot L_{M}}{T} \cdot \frac{\left(V_{2}-V_{1}\right) \cdot\left(I_{1} \cdot V_{1}+I_{2} \cdot V_{2}\right)}{V_{g} \cdot\left(V_{g}+V_{1}\right) \cdot\left(V_{g}+V_{2}\right)} \\
V_{1} & =\frac{n_{P}}{n_{1}} \cdot V_{O 1}, V_{2}=\frac{n_{P}}{n_{2}} \cdot V_{O 2}, \\
I_{1} & =\frac{n_{1}}{n_{P}} \cdot I_{O 1}, I_{2}=\frac{n_{2}}{n_{P}} \cdot I_{O 2}
\end{aligned}
$$


To solve the root $d$ of the third-order (5), one may follow the procedure in the Appendix (Section 7). As soon as $d$ is found, it would be easy to find $d_{1}$ and $d_{2}$ from (2) and (3). Figure 3 shows the relations of duty cycles versus load conditions that are applied to the example in Section 4.

Since it is hard to find a root of the third-order equation or a higher-order equation, approximation could be made by assuming that the current ripples are negligible. The approximate equation can be obtained from (4) by neglecting the last item

$$
\frac{I_{P}}{d}=\frac{I_{1}}{d_{1}}=\frac{I_{2}}{d_{2}}=I_{M A}
$$

where $I_{M A}$ is the average magnetising current of transformer.

From (6), we find that the relations between duties and input/output depend on the average currents. This is a very interesting feature. In most topologies of switching mode power supplies, the relations are dominated by voltages rather than currents.

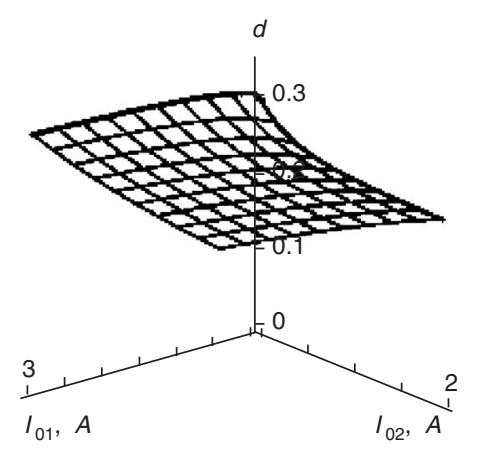

$a$

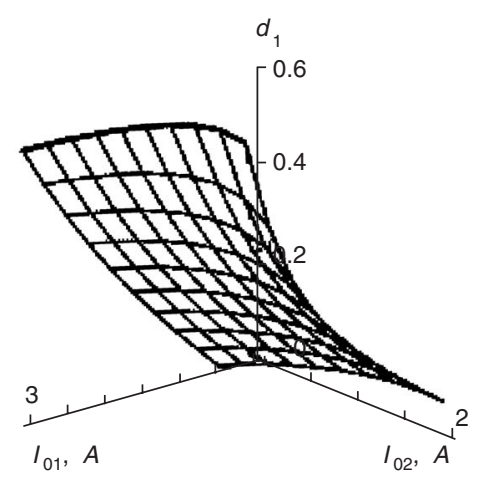

$b$

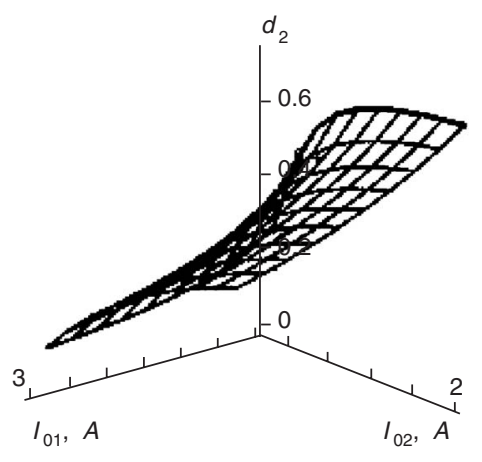

C

Fig. 3 Duty cycles plotted against load conditions $V_{g}=20 \mathrm{~V}, V_{O 1}=3.3 \mathrm{~V}, V_{O 2}=5 \mathrm{~V}, I_{O 1}=0-3 \mathrm{~A}, I_{O 2}=0-2 \mathrm{~A}$ $a$ duty $d$

$b$ duty $d_{1}$

$c$ duty $d_{2}$

\section{Analytical model with nonideal characteristics of components}

In practical design, the performance of the flyback converter with magamp application is limited by the nonideal characteristics of the components. Since the energy delivery for each output depends on the current sharing principle, some parasitic parameters such as leakage inductance and saturated inductance may limit the rate of current flow. These will not only constrain the number of outputs, but also the operating area under extreme load conditions.

An analytical model is now proposed to explore the internal mechanism and to investigate the critical factors that affect proper operation.

\subsection{Circuit analysis of proposed analytical model}

To simplify the analysis, some reasonable assumptions are made: first, when the main switch and the diodes are turned on, they are considered as short circuits. The voltage drop of onresistance (Rds,on) of the main switch is negligible compared with the input voltage $V_{g}$. In the low output voltage application, the drops of the diodes could be lumped with the output voltages. Under the off state, they are represented as an open circuit.

Secondly, the main transformer can be represented as an ideal transformer with a magnetising inductance $L_{M}$ and equivalent leakage inductances, $L_{K P}, L_{K 1}$ and $L_{K 2}$, corresponding to primary winding, secondary winding 1 and secondary winding 2 , respectively. The voltage drops of the winding resistances are small enough compared with the input voltage $V_{g}$ and output $V_{O 1}$ and $V_{O 2}$. They are neglected in the model.

Thirdly, the output capacitances $C_{1}, C_{2}$ and the capacitance of the clamp snubber $C_{C}$ are sufficiently large that the voltages across these capacitances could be considered to be constant during the switching cycle.

Finally, the behaviour of the saturable reactor $S R$ can be modelled as an inductance $L_{S R}$ when saturated. During block operation, it could be viewed as an open circuit. The core loss due to the hysteresis characteristics is sufficiently small so that it can be neglected.

The equivalent circuit model of Fig. 1 is shown in Fig. 4 where all secondary quantities have been referred to the primary side. A typical RC clamp snubber circuit is added on the primary side to prevent high voltage stress due to the energy stored in the leakage inductance $L_{K P}$.

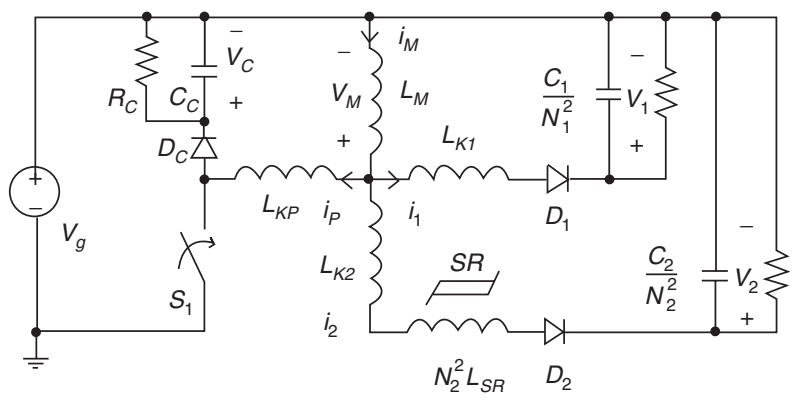

Fig. 4 Equivalent circuit model of Fig. 1

Figure $5 a$ shows the key waveforms that are simulated by the proposed equivalent model in normal continuous current mode. It would be easy to indicate that some current transition periods are inserted among every duty period in the previous ideal case. In particular, the time 

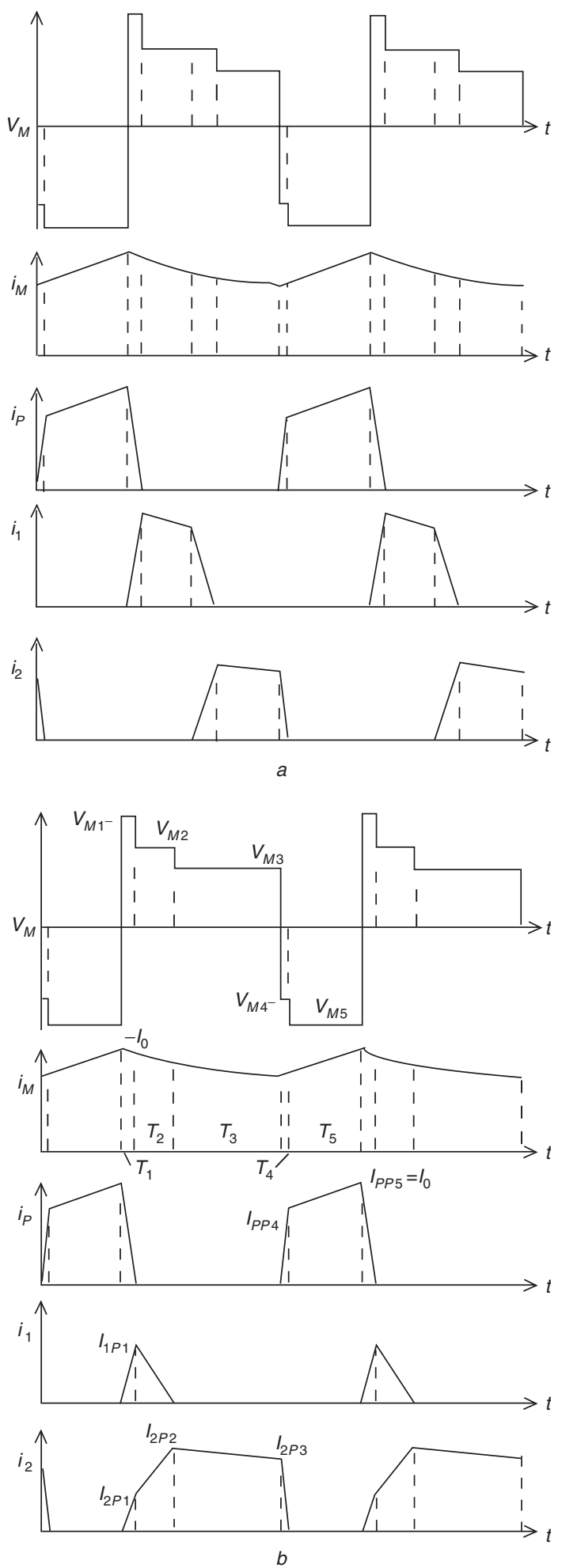

Fig. 5 Key waveforms that are simulated by the proposed equivalent model

$a$ normal condition

$b$ boundary condition

interval in which diodes $D_{1}$ and $D_{2}$ are on is the most evident in real applications and deserves attention. As the load of output 2 increases the duty for output 2 also increases but the blocking time of the saturable inductor decreases. Under the extreme load condition, the blocking time becomes a minimum. Since the current rise time is limited by the leakage inductance and the saturated inductance, output 2 cannot obtain enough energy and becomes nonoperational. Figure $5 b$ shows the key waveforms under extreme load conditions.
3.1.1 $T_{1}$ time interval: At the beginning of the $T_{1}$ time interval, the main switch $S_{1}$ is turned off. The magnetising current starts to charge outputs 1 and 2 . At the same time the energy stored in the leakage inductance $L_{K P}$ is absorbed by the capacitor of the RC clamp snubber. The equivalent circuit during the $T_{1}$ interval is represented as that shown in Fig. $6 a$. Thus, one can obtain the following equations:

$$
\begin{gathered}
i_{M}=i_{P}+i_{1}+i_{2} \\
i_{M}(t)=-\frac{V_{M 1}}{L_{M}} \cdot t+I_{0} \\
i_{P}(t)=\frac{\left(V_{M 1}-V_{C}\right) K_{P}}{L_{M}} \cdot t+I_{0} \\
i_{1}(t)=\frac{\left(V_{M 1}-V_{1}\right) K_{1}}{L_{M}} \cdot t \\
i_{2}(t)=\frac{\left(V_{M 1}-V_{2}\right) K_{2}}{L_{M}} \cdot t
\end{gathered}
$$

Substituting (8)-(11) into (7), we obtain

$$
\begin{aligned}
-V_{M 1}= & \left(V_{M 1}-V_{C}\right) K_{P} \\
& +\left(V_{M 1}-V_{1}\right) K_{1}+\left(V_{M 1}-V_{2}\right) K_{2}
\end{aligned}
$$

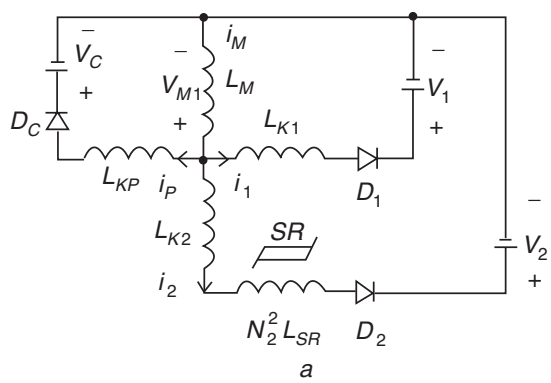

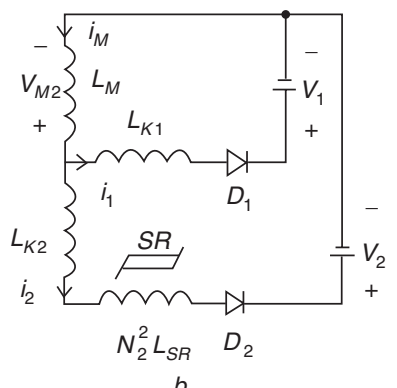

b

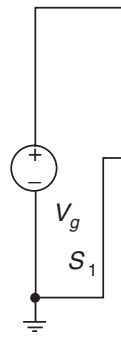

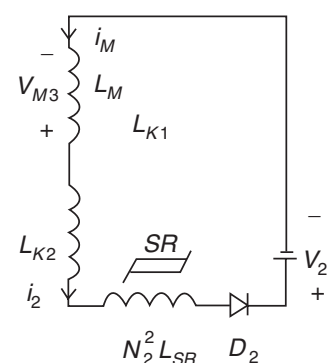

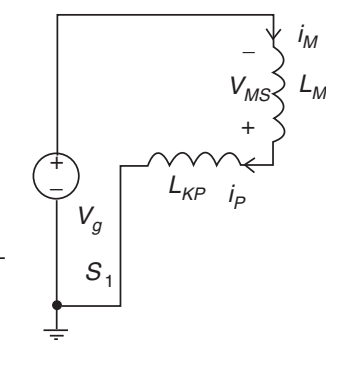

Fig. 6 Equivalent circuit for different time intervals a $T_{1}$ time interval $b T_{2}$ time interval $c T_{3}$ time interval $d T_{4}$ time interval e $T_{5}$ time interval 
Solving (12) for $V_{M 1}$ yields

$$
V_{M 1}=\frac{K_{1} V_{1}+K_{2} V_{2}+K_{P} V_{C}}{1+K_{1}+K_{2}+K_{P}}
$$

At the end of the $T_{1}$ interval, $T_{1}$ is obtained from (9) by letting $i_{P}\left(T_{1}\right)=0$.

$$
T_{1}=\frac{L_{M} I_{0}}{K_{P}} \cdot \frac{1+K_{1}+K_{2}+K_{P}}{V_{C}+K_{1} V_{C}+K_{2} V_{C}-K_{1} V_{1}-K_{2} V_{2}}
$$

Substituting $T_{1}$ and $V_{M 1}$ into (10) and (11), the peak currents of $i_{1}$ and $i_{2}$ at the end of $T_{1}$ are, respectively

$$
\begin{aligned}
& I_{1 P 1}=\frac{K_{1}}{K_{P}} \cdot \frac{K_{2} V_{2}+K_{P} V_{C}-V_{1}-K_{2} V_{1}-K_{P} V_{1}}{V_{C}+K_{1} V_{C}+K_{2} V_{C}-K_{1} V_{1}-K_{2} V_{2}} \cdot I_{0} \\
& I_{2 P 1}=\frac{K_{2}}{K_{P}} \cdot \frac{K_{1} V_{1}+K_{P} V_{C}-V_{2}-K_{1} V_{2}-K_{P} V_{2}}{V_{C}+K_{1} V_{C}+K_{2} V_{C}-K_{1} V_{1}-K_{2} V_{2}} \cdot I_{0}
\end{aligned}
$$

In this period, the energy stored in the leakage $L_{K P}$ had been transferred to the RC clamp snubber. Assuming the voltage $V_{C}$ is constant, the energy will be dissipated by the resistance $R_{C}$. The total power loss of snubber can be derived as follows:

$$
\begin{aligned}
P_{R C} & =\frac{1}{T} \int_{0}^{T_{1}} V_{C} \cdot i_{P}(t) \cdot d t \\
& =\frac{L_{M} I_{0}^{2}}{2 T} \cdot \frac{\left(1+K_{1}+K_{2}+K_{P}\right) V_{C}}{K_{P}\left(V_{C}+K_{1} V_{C}+K_{2} V_{C}-K_{1} V_{1}-K_{2} V_{2}\right)}=\frac{V_{C}^{2}}{R_{C}}
\end{aligned}
$$

Solving (17), the voltage of the snubber capacitor $C_{C}$ is

$$
V_{C}=\frac{\left(K_{1} V_{1}+K_{2} V_{2}\right)+\sqrt{\left(K_{1} V_{1}+K_{2} V_{2}\right)^{2}+\frac{2 L_{M} R_{C} I_{0}^{2}\left(1+K_{1}+K_{2}+K_{P}\right)\left(1+K_{1}+K_{2}\right)}{T \cdot K_{P}}}}{2\left(1+K_{1}+K_{2}\right)}
$$

3.1.2 $T_{2}$ time interval: As the current of $L_{K P}$ decreases to zero, the diode $D_{C}$ is reverse biased and the operation enters the $T_{2}$ time interval. The equivalent circuit model is given in Fig. $6 b$. Similarly, the following equations can be obtained:

$$
\begin{gathered}
i_{M}=i_{1}+i_{2} \\
i_{M}(t)=-\frac{V_{M 2}}{L_{M}} \cdot t+I_{1 P 1}+I_{2 P 1} \\
i_{1}(t)=\frac{\left(V_{M 2}-V_{1}\right) K_{1}}{L_{M}} \cdot t+I_{1 P 1} \\
i_{2}(t)=\frac{\left(V_{M 2}-V_{2}\right) K_{2}}{L_{M}} \cdot t+I_{2 P 1}
\end{gathered}
$$

Similarly, $V_{M 2}$ can be found as

$$
V_{M 2}=\frac{K_{1} V_{1}+K_{2} V_{2}}{1+K_{1}+K_{2}}
$$

$T_{2}$ is also obtained from (21) by letting $i_{1}\left(T_{2}\right)=0$.

$$
\begin{aligned}
T_{2}= & \frac{L_{M} I_{0}}{K_{P}} \cdot \frac{1+K_{1}+K_{2}}{V_{1}+K_{2} V_{1}-K_{2} V_{2}} \\
& \cdot \frac{K_{2} V_{2}+K_{P} V_{C}-V_{1}-K_{2} V_{1}-K_{P} V_{1}}{V_{C}+K_{1} V_{C}+K_{2} V_{C}-K_{1} V_{1}-K_{2} V_{2}}
\end{aligned}
$$

Substituting (16), (23) and (24) into (22), we can obtain the peak current $I 2 P 2$

$$
I_{2 P 2}=\frac{K_{2}\left(V_{1}-V_{2}\right)}{V_{1}+K_{2} V_{1}-K_{2} V_{2}} \cdot I_{0}
$$

3.1.3 $T_{3}$ time interval: When the current of diode $D_{2}$ decays to zero, the $T_{3}$ time interval begins. Only $L_{M}$ and $L_{K 2}$ have current through it. The equivalent circuit model during $T_{3}$ is shown in Fig. $6 c$. Similarly to the $T_{1}$ and $T_{2}$ intervals, we have

$$
\begin{gathered}
i_{M}=i_{2} \\
i_{M}(t)=-\frac{V_{M 3}}{L_{M}} \cdot t+I_{2 P 2} \\
i_{2}(t)=\frac{\left(V_{M 3}-V_{2}\right) K_{2}}{L_{M}} \cdot t+I_{2 P 2}
\end{gathered}
$$

Solving (26)-(28), $V_{M 3}$ can be derived as

$$
V_{M 3}=\frac{K_{2} V_{2}}{1+K_{2}}
$$

Substituting (25) and (29) into (28), the peak current of $i_{2}$ at the end of $T_{3}$ is

$$
I_{2 P 3}=\frac{-K_{2} V_{2}}{L_{M}\left(1+K_{2}\right)} \cdot T_{3}+\frac{K_{2}\left(V_{1}-V_{2}\right)}{V_{1}+K_{2} V_{1}-K_{2} V_{2}} \cdot I_{0}
$$

3.1.4 $T_{4}$ time interval: At the beginning of the $T_{4}$ interval, the main switch turns on again and then the input starts to charge $L_{M}$. Figure $6 d$ shows the equivalent circuit model in the $T_{4}$ interval. As in the previous process, we have

$$
\begin{gathered}
i_{M}=i_{P}+i_{2} \\
i_{M}(t)=-\frac{V_{M 4}}{L_{M}} \cdot t+I_{2 P 3} \\
i_{P}(t)=\frac{\left(V_{M 4}+V_{g}\right) K_{P}}{L_{M}} \cdot t \\
i_{2}(t)=\frac{\left(V_{M 4}-V_{2}\right) K_{2}}{L_{M}} \cdot t+I_{2 P 3}
\end{gathered}
$$

Solving (31)-(34), $V_{M 4}$ is

$$
V_{M 4}=\frac{K_{2} V_{2}-K_{P} V_{g}}{1+K_{2}+K_{P}}
$$

$T_{4}$ is obtained from (34) by letting $i_{2}\left(T_{4}\right)=0$.

$$
\begin{aligned}
T_{4}= & \frac{L_{M}}{K_{2}} \cdot \frac{1+K_{2}+K_{P}}{V_{2}+K_{P} V_{2}+K_{P} V_{g}} \cdot\left(\frac{-K_{2} V_{2}}{L_{M}\left(1+K_{2}\right)} \cdot T_{3}\right. \\
& \left.+\frac{K_{2}\left(V_{1}-V_{2}\right)}{V_{1}+K_{2} V_{1}-K_{2} V_{2}} \cdot I_{0}\right)
\end{aligned}
$$

And the peak current of $i_{P}$ at the end of $T_{4}$ can be found by substituting (35) and (36) into (33)

$$
\begin{aligned}
I_{P P 4}= & \frac{K_{P}}{K_{2}} \cdot \frac{V_{g}+K_{2} V_{g}+K_{2} V_{2}}{V_{2}+K_{P} V_{2}+K_{P} V_{g}} \cdot\left(\frac{-K_{2} V_{2}}{L_{M}\left(1+K_{2}\right)} \cdot T_{3}\right. \\
& \left.+\frac{K_{2}\left(V_{1}-V_{2}\right)}{V_{1}+K_{2} V_{1}-K_{2} V_{2}} \cdot I_{0}\right)
\end{aligned}
$$

3.1.5 $T_{5}$ time interval: As long as the current $i_{2}$ drops to zero, the diode $D_{2}$ is off and the current loop through $L_{M}$ and $L_{K P}$ only exists on the primary side. The equivalent circuit model is presented in Fig. 6e. Similarly, one may obtain

$$
\begin{gathered}
i_{M}=i_{P} \\
i_{M}(t)=-\frac{V_{M 5}}{L_{M}} \cdot t+I_{P P 4}
\end{gathered}
$$




$$
i_{P}(t)=\frac{\left(V_{M 5}+V_{g}\right) K_{P}}{L_{M}} \cdot t+I_{P P 4}
$$

Solving (38)-(40) for $V_{M 5}$ yields

$$
V_{M 5}=\frac{-K_{P} V_{g}}{1+K_{P}}
$$

Substituting (37) and (41) into (40), the peak current $I_{P P 5}$ is expressed by

$$
\begin{array}{r}
I_{P P 5}=\frac{K_{P} V_{g}}{L_{M}\left(1+K_{P}\right)} \cdot T_{5}+\frac{K_{P}}{K_{2}} \cdot \frac{V_{g}+K_{2} V_{g}+K_{2} V_{2}}{V_{2}+K_{P} V_{2}+K_{P} V_{g}} \\
\cdot\left[\frac{-K_{2} V_{2}}{L_{M}\left(1+K_{2}\right)} \cdot T_{3}+\frac{K_{2}\left(V_{1}-V_{2}\right)}{V_{1}+K_{2} V_{1}-K_{2} V_{2}} \cdot I_{0}\right]
\end{array}
$$

Due to the continuity of the inductor current in steady state operation, the following equation is satisfied:

$$
I_{P P 5}=I_{0}
$$

Substituting (43) into (42), one can obtain

$$
T_{5}-A \cdot T_{3}=B \cdot I_{0}
$$

where

$$
\begin{aligned}
A & =\frac{V_{2}\left(1+K_{P}\right)\left(V_{g}+K_{2} V_{g}+K_{2} V_{2}\right)}{V_{g}\left(1+K_{2}\right)\left(V_{2}+K_{P} V_{2}+K_{P} V_{g}\right)} \\
B & =\frac{L_{M} V_{2}\left(1+K_{P}\right)\left(V_{1}+K_{2} V_{1}-K_{2} V_{2}+K_{P} V_{1}+K_{P} V_{g}\right)}{K_{P} V_{g}\left(V_{2}+K_{P} V_{2}+K_{P} V_{g}\right)\left(V_{1}+K_{2} V_{1}-K_{2} V_{2}\right)}
\end{aligned}
$$

For a switching period $T$, we have

$$
T=T_{1}+T_{2}+T_{3}+T_{4}+T_{5}
$$

Substituting (14), (24) and (36) into (45) yields

$$
T_{5}+C \cdot T_{3}=T-D \cdot I_{0}
$$

where

$$
\begin{aligned}
& C=\frac{K_{P}\left(V_{g}+K_{2} V_{g}+K_{2} V_{2}\right)}{\left(1+K_{2}\right)\left(V_{2}+K_{P} V_{2}+K_{P} V_{g}\right)} \\
& D=\frac{L_{M}\left(V_{1}+K_{2} V_{1}-K_{2} V_{2}+K_{P} V_{1}+K_{P} V_{g}\right)}{\left(V_{2}+K_{P} V_{2}+K_{P} V_{g}\right)\left(V_{1}+K_{2} V_{1}-K_{2} V_{2}\right)}
\end{aligned}
$$

$T_{3}$ and $T_{5}$ can be obtained by solving (44) and (46)

$$
\begin{gathered}
T_{3}=\frac{V_{g}\left(1+K_{2}\right)}{V_{g}+K_{2} V_{g}+K_{2} V_{2}} \cdot T-\frac{L_{M}\left(1+K_{2}\right)}{K_{P}} \\
\cdot \frac{\left(V_{1}+K_{2} V_{1}-K_{2} V_{2}+K_{P} V_{1}+K_{P} V_{g}\right)}{\left(V_{g}+K_{2} V_{g}+K_{2} V_{2}\right)\left(V_{1}+K_{2} V_{1}-K_{2} V_{2}\right)} \cdot I_{0} \\
T_{5}=\frac{V_{2}\left(1+K_{P}\right)}{V_{2}+K_{P} V_{2}+K_{P} V_{g}} \cdot T
\end{gathered}
$$

\subsection{Analytical expression of boundary condition}

Based on the above analysis, the average currents of outputs 1 and 2 under the boundary condition are calculated as follows:

$$
\begin{aligned}
I_{1 A}= & \frac{1}{2 T} \cdot I_{1 P 1} \cdot\left(T_{1}+T_{2}\right) \\
= & \frac{K_{1} L_{M} I_{0}^{2}}{2 K_{P} T} \\
& \cdot \frac{\left(K_{2} V_{2}+K_{P} V_{C}-V_{1}-K_{2} V_{1}-K_{P} V_{1}\right)}{\left(V_{C}+K_{1} V_{C}+K_{2} V_{C}-K_{1} V_{1}-K_{2} V_{2}\right)\left(V_{1}+K_{2} V_{1}-K_{2} V_{2}\right)}
\end{aligned}
$$

$$
\begin{aligned}
I_{2 A} & =\frac{1}{2 T} \cdot\left[I_{2 P 1}\left(T_{1}+T_{2}\right)+I_{2 P 2}\left(T_{2}+T_{3}\right)+I_{2 P 3}\left(T_{3}+T_{4}\right)\right] \\
& =E \cdot I_{1 A}+\frac{1}{2 T} \cdot\left[I_{2 P 2}\left(T_{2}+T_{3}\right)+I_{2 P 3}\left(T_{3}+T_{4}\right)\right]
\end{aligned}
$$

where

$$
E=\frac{K_{2}\left(K_{1} V_{1}+K_{P} V_{C}-V_{2}-K_{1} V_{2}-K_{P} V_{2}\right)}{K_{1}\left(K_{2} V_{2}+K_{P} V_{C}-V_{1}-K_{2} V_{1}-K_{P} V_{1}\right)}
$$

The solution of (50) can be obtained easily by substituting each individual item that has already been derived before. However, it is trivial and too complex to analysis the relation between $I_{1 A}$ and $I_{2 A}$. To obtain an analytical expression of the boundary condition, some reasonable assumptions could be made. First, since $T_{2}$ and $T_{4}$ are transition periods, they could be neglected compared with $T_{3}$. Secondly, the magnetising inductance $L_{M}$ is large enough, so that the can decay can be neglected during $T_{3}$, i.e. $I_{2 P 2} \approx I_{2 P 3} \approx I_{0}$. Finally, the leakage inductances $L_{K 1}$, $L_{K 2}, L_{K P}$ and the saturated inductance $L_{S R}$ are much smaller than the magnetising inductance $L_{M}$, such that $K_{1}$, $K_{2}$ and $K_{P}>>1$.

Equation (50) can be approximated by

$$
I_{2 A} \approx E \cdot I_{1 A}+\frac{I_{0} \cdot T_{3}}{T}
$$

Equation (47) can be also approximated by

$$
\begin{aligned}
T_{3} \approx & \frac{V_{g}}{V_{g}+V_{2}} \cdot T \\
& -I_{0}\left[\frac{L_{K P}}{V_{g}+V_{2}}+\frac{\left(L_{K 2}+N_{2}^{2} L_{S R}\right)\left(V_{g}+V_{1}\right)}{\left(V_{1}-V_{2}\right)\left(V_{g}+V_{2}\right)}\right]
\end{aligned}
$$

Substituting (52) into (51) yields

$$
\begin{aligned}
I_{2 A} \approx & E \cdot I_{1 A}+\frac{V_{g}}{V_{g}+V_{2}} I_{0} \\
& -\frac{I_{0}^{2}}{T}\left[\frac{L_{K P}}{V_{g}+V_{2}}+\frac{\left(L_{K 2}+N_{2}^{2} L_{S R}\right)\left(V_{g}+V_{1}\right)}{\left(V_{1}-V_{2}\right)\left(V_{g}+V_{2}\right)}\right]
\end{aligned}
$$

Equation (53) will help us to understand the factors that affect the boundary condition in Section 4 .

\section{Experimental results}

To illustrate the effectiveness of the previous analysis, some experimental results are given below. A $20 \mathrm{~W}$ two-output flyback converter is constructed having the following parameters:

$\begin{array}{ll}\text { input range } V_{g} & 20-30 \mathrm{~V} \\ \text { output } V_{O 1} & 3.3 \mathrm{~V} / 0-3 \mathrm{~A} \\ \text { output } V_{O 2} & 5 \mathrm{~V} / 0-2 \mathrm{~A} \\ \text { switching period } T & 10 \mu \mathrm{S} \\ \text { turn number } n_{P} / n_{1} / n_{2} & 10 / 3 / 8 \\ \text { leakage inductance } L_{K P} / L_{K 1} / L_{K 2} 2 & 0.95 / 0.96 / 0.94 \mu \mathrm{H} \\ \text { magnetising inductance } L_{M} & 70 \mu \mathrm{H} \\ \text { saturated inductance } L_{S R} & 0.45 \mu \mathrm{H}\end{array}$

The core number of the main transformer is EI33 from TDK corporation. The saturable reactor has eight turns and the core number is MP1506 from Allied Signal. The common used current reset scheme is applied to reset saturable reactor. The clamp snubber capacitance and resistance are $0.1 \mathrm{uF}$ and $1 \mathrm{k} \Omega$, respectively. The output diode $D_{1}$ is a Schottky diode SBL840 from Transys Electronics Limited and the diode $D_{2}$ is a fast diode SF1004G from Taiwan Semiconductor 
Co. Ltd. Since both output voltages are low, the voltage drops of diodes are taken into account in the simulations. The voltage drops of $D_{1}$ and $D_{2}$ under boundary load conditions are $0.1 \mathrm{~V}$ and $0.9 \mathrm{~V}$, respectively.

Figure 7 shows some experimental waveforms under: (a) half load; (b) full load; (c) boundary load conditions. In Fig. 7, $V_{P}$ is the voltage across the primary winding of the transformer as shown in Fig. 1 and $i_{O 1}, i_{O 2}$ are the instantaneous currents of outputs 1 and 2, respectively. Figure 8 shows the boundary condition for the average output currents $I_{O 1}$ and $I_{O 2}$ with both the analytical model and experimental results. It is obvious that the experimental results are in good agreement with the proposed analytical model. We can find the boundary conditions when the output current $I_{O_{2}}$ is a heavy load and $I_{O 1}$ is a light load. On the left side of the boundary line, output 2 becomes out of regulation. The results also verify the effectiveness of (50). However, (50) is complex and trivial. The simplified (53) is more comprehensive and analytical. In (53), output currents $I_{1 A}$ and $I_{2 A}$ are a function of $I_{0}$ which depends on the load condition. The only nonideal factors that affect the boundary (53) are the leakage inductances $L_{K P}, L_{K 2}$ and

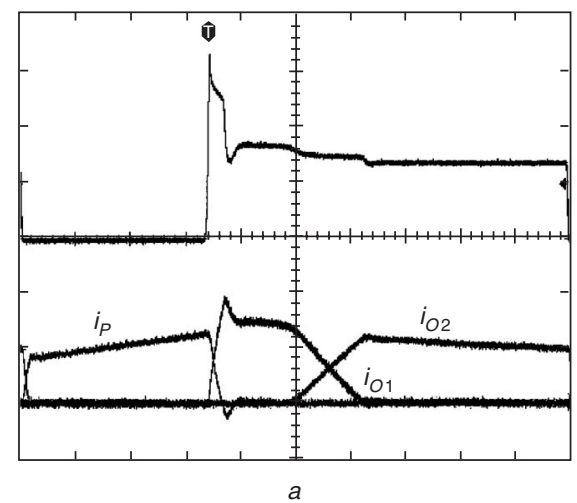

$V_{p}, 20 \mathrm{~V} / \mathrm{div}$

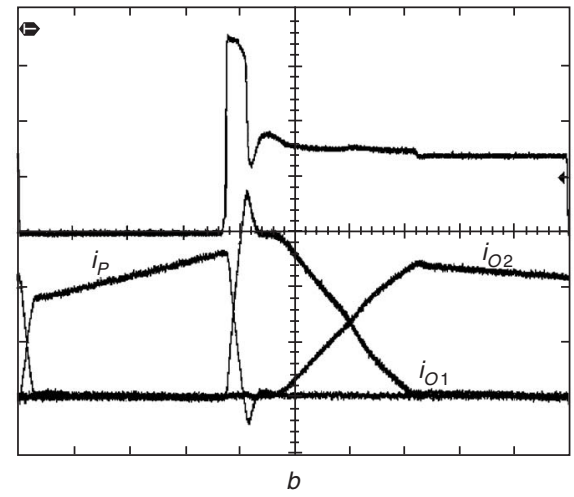

$V_{p}, 20 \mathrm{~V} / \mathrm{div}$

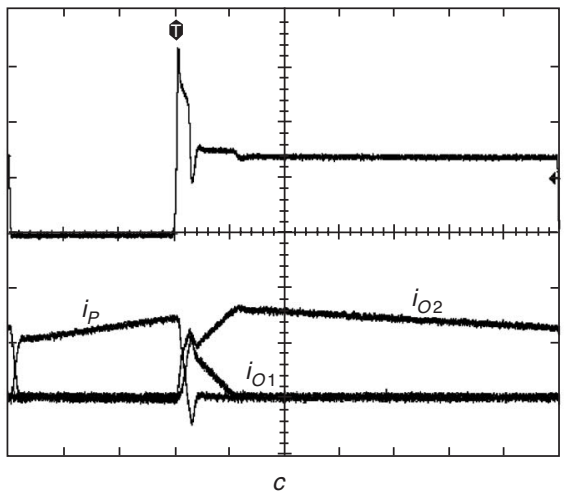

$V_{p}, 20 \mathrm{~V} / \mathrm{div}$

Fig. 7 Some experimental waveforms time scale is $1 \mu \mathrm{s} /$ div $a$ half load: $V_{g}=20 \mathrm{~V}, I_{O 1}=3 \mathrm{~A}, I_{O 2}=2 \mathrm{~A}$

$b$ full load: $V_{g}=20 \mathrm{~V}, I_{O 1}=1.5 \mathrm{~A}, I_{O 2}=1 \mathrm{~A}$

$c$ boundary load: $V_{g}=20 \mathrm{~V}, I_{O 1}=0.249 \mathrm{~A}, I_{O 2}=2 \mathrm{~A}$
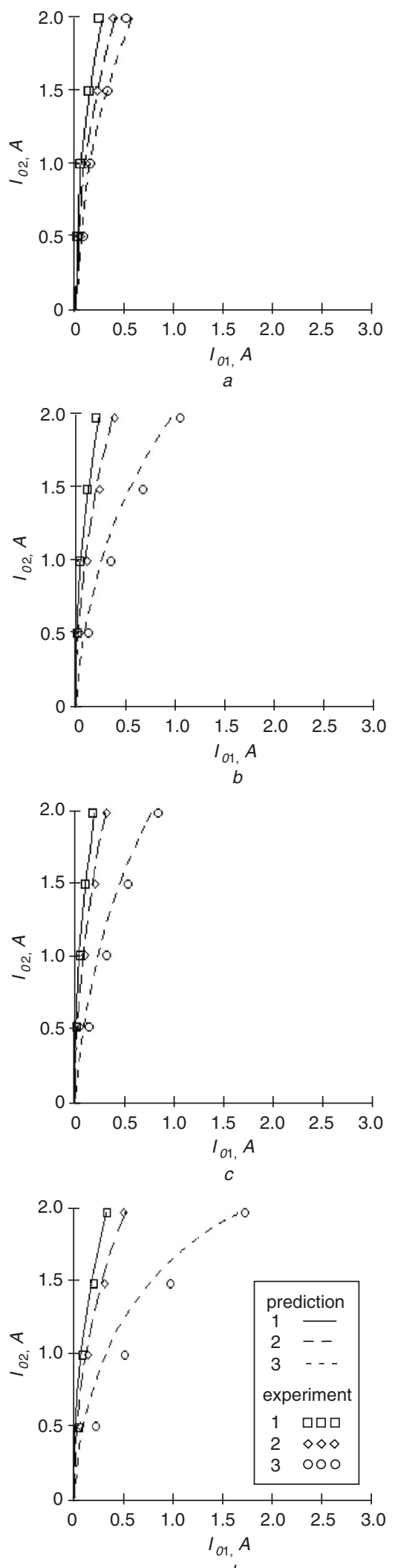

Fig. 8 Experimental results with different design conditions $a$ under $V_{g}=20 \mathrm{~V}, T=10 \mu \mathrm{s}$ and $n_{2}=8 \mathrm{~T}$ condition: $1 L_{S R}=0.45 \mu \mathrm{H}, 2 L_{S R}=0.7 \mu \mathrm{H}, 3 L_{S R}=1 \mu \mathrm{H}$ $b$ under $V_{g}=20 \mathrm{~V}, T=10 \mu \mathrm{s}$ and $L_{S R}=0.45 \mu \mathrm{H}$ condition: $1 n_{2}=8 \mathrm{~T}, 2 n_{2}=7 \mathrm{~T}, 3 n_{2}=6 \mathrm{~T}$ $c$ under $V_{g}=30 \mathrm{~V}, T=10 \mu \mathrm{s}$ and $L_{S R}=0.45 \mu \mathrm{H}$ condition: $1 n_{2}=8 \mathrm{~T}, 2 n_{2}=7 \mathrm{~T}, 3 n_{2}=6 \mathrm{~T}$ $d$ under $V_{g}=20 \mathrm{~V}, T=6.67 \mu \mathrm{s}$ and $L_{S R}=0.45 \mu \mathrm{H}$ condition: $1 n_{2}=8 \mathrm{~T}, 2 n_{2}=7 \mathrm{~T}, 3 n_{2}=6 \mathrm{~T}$ 
the saturated inductance $L_{S R}$. The current rise time is limited by these factors. Hence, the magnetising inductance $L_{M}$ cannot supply enough energy to output 2 beyond the boundary. The other factors of (53) are designed parameters such as the switching period $T$, input voltage $V_{g}$, output voltages $V_{1}, V_{2}$, turn ratio $N_{2}$ and the turns of the saturable reactor. Figure $8 a-d$ show the effectiveness of the designed factors with different values compared to the original design.

In Fig. $8 a$, three different turns of saturable reactor are provided: condition 1 is the original value: 8 turns and $L_{S R}=0.45 \mu \mathrm{H}$, condition 2 is 10 turns and $L_{S R}=0.7 \mu \mathrm{H}$ and condition 3 is 12 turns and $L_{S R}=1 \mu \mathrm{H}$. It can be seen that the boundary lines move toward the right with increasing turns of the saturable reactor. This complies with the conclusion of (53) that the larger the leakage inductances and the saturated inductance are, the worse the boundary condition is. It seems that fewer turns of the saturable reactor are preferred. However, the side effects should be considered to reduce the saturated inductance. More reset current from the controller is needed when the turns of the saturable reactor are decreased. On the other hand, it reduces the volt-second blocking range of the saturable inductor.

Since the output voltages $V_{O 1}$ and $V_{O 2}$ are defined by the specification, the actual designed factors are the turn ratios for $V_{1}$ and $V_{2}$. The turn ratio $N_{1}$ for the main output $V_{1}$ can be designed by the traditional approach. However, the turn ratio $N_{2}$ for the magamp output $V_{2}$ becomes a critical factor affecting the boundary. Figure $8 b$ shows the effect with three different turns for winding 2 i.e. condition 1 is the original value: $n_{2}=8$ turns, condition $2: n_{2}=7$ turns and condition 3: $n_{2}=6$ turns. As the winding turns of output 2 decrease, the voltage difference between $V_{1}$ and $V_{2}$ will be reduced. It can be observed from (53) that the third item in the right-hand side of the equation will be increased and the boundary lines are shifted to the right. The major reason is that reducing the voltage difference $V_{1}-V_{2}$ across $L_{K 2}$ will increase the current rise time. This factor becomes significant when the voltage difference is too small. To prevent this, one should increase the winding turns. However, this will increase the voltage stress on the output diode.

As the input voltage $V_{g}$ is changed to a high line voltage $\left(V_{g}=30 \mathrm{~V}\right)$, all the boundary lines with the same conditions as in Fig. $8 b$ are shifted to the left. This is because higher input voltage can speed up the current rise time. The effect is shown in Fig. $8 c$. This also means that the worse case condition is under the low line condition.

In Fig. $8 d$, the switching frequency is increased to $150 \mathrm{kHz}$, i.e. the switching period $(T=6.67 \mu \mathrm{S})$ becomes two-thirds of the original value. The boundary lines with the same conditions as in Fig. $8 b$ are moved further towards the right. This is because the time period for the current distribution is further limited. This can be also explained by (53), that decreasing the switching period will also be increase the third item.

From the above discussions, the design becomes a tradeoff problem. One should design the parameters carefully to obtain the optimum performance. However, under any condition, the minimum load requirement on output 1 is necessary to keep regulation. A simple and cost effective way to solve this issue is to add a preload on output 1 . In this example, a $1 \mathrm{~W}$ preload is placed to meet the $1 \%$ regulation requirement. Figure 9 shows the cross-regulation of output $V_{O 1}$ and $V_{O 2}$ (without and with magamp, respectively). Output $V_{O 1}$ is controlled by the PWM feedback loop. The cross-regulation of output $V_{O 1}$ meets
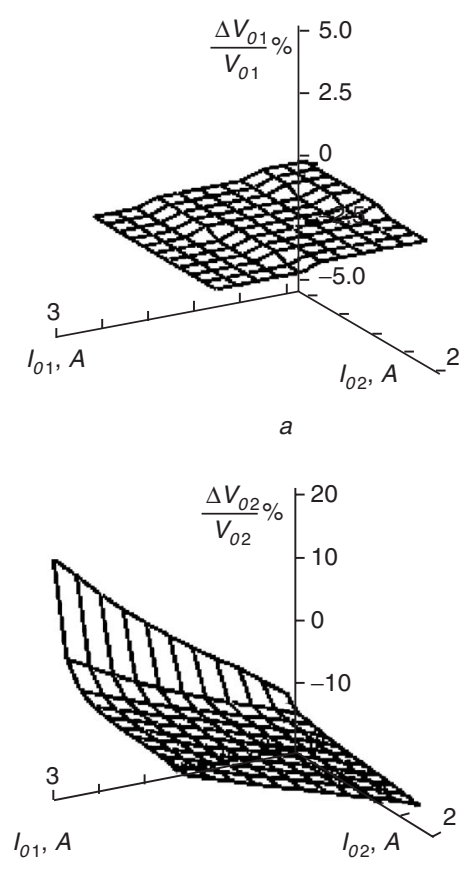

$b$

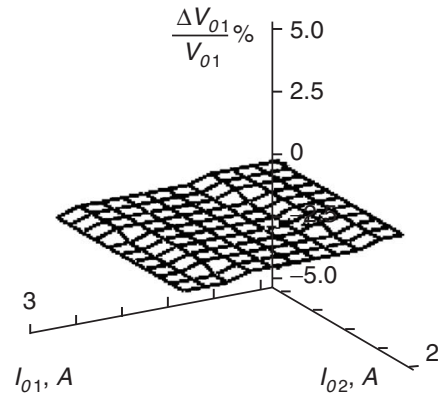

$c$

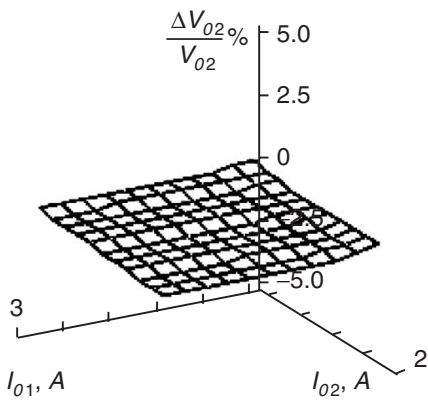

Fig. 9 Cross-regulation of output $V_{O 1}, V_{O 2}$ $a$ output $V_{O 1}$ without magamp $b$ output $V_{O_{2}}$ without magamp $c$ output $V_{O 1}$ with magamp $d$ output $V_{O 2}$ with magamp

the $1 \%(0.3-0.3 \%)$ regulation requirement for with and without magamp condition. On the other hand, the crossregulation of output $V_{O 2}$ without magamp control is varied from $16.9-16.9 \%$. As the saturable reactor is added and the magamp feedback loop is enabled, the cross-regulation of output $V_{O 2}$ is well under $1 \%(0.4-0.4 \%)$ regulation requirement.

\section{Conclusions}

We have investigated the operation of the magamp postregulator in flyback converters with multiple output 
windings. The circuit of the magamp regulator looks similar to that of the forward converters. But the operating principle is totally different. The output of the main transformer in the flyback converter is viewed as a current source. The energy is supplied to each output winding by the time-sharing approach. Due to the leakage inductances and the saturated inductance, the current rise time is limited so that some transition periods are inserted. The proposed analytical model effectively explains the mechanism of the regulation boundary. The experimental results matched the predicted boundary conditions. A simple analytical expression is derived to explain the factors that affect the boundary lines, such as: leakage inductance, saturated inductance, switching frequency and input voltage. To maintain the regulation, a minimum load is required. Finally, the experimental example shows the effective of applying the magamp in the flyback converter. Both output regulations are excellent with a $1 \mathrm{~W}$ preload on output 1 .

\section{References}

1 Chen, Q., Lee, F.C., and Jovanovic, M.M.: 'Analysis and design of weighted voltage-mode control for a multiple-output forward converter'. APEC, San Diego, CA, USA, 1993, pp. 449-455

2 Maksimovic, D., and Erickson, R.: 'Modeling of cross regulation in multiple-output flyback converters'. APEC, Dallas, TX, USA, 1999, pp. $350-356$

3 Ji, C., Smith, K.M., Smedley, K.M., and King, K.: 'Cross regulation in flyback converter: analytic model and solution', IEEE Trans. Power Electron., 2001, 16, (2), pp. 231-239

4 Kotlarewsky, P.: 'Beyond the limitation of reset control and square loop of materials for mag-amp post regulators'. High Frequency Power Conversion Conference, Toronto, Canada, 1991, pp. 129-139

5 Lee, J., Chen, D.Y., and Jamerson, C.: 'Magamp post regulators-practical design considerations to allow operation under extreme loading conditions', IEEE Trans. Power Electron., 1990, 5, (1), pp. 69-76

6 Tedder, R.M.: 'Effects of converter type, reset method and core material on magamp regulator performance'. APEC, Baltimore, MD, USA, 1989, pp. 391-400

7 Nelson, O.N.: 'Time share mag amp'. High Frequency Power Conversion Conference, San Diego, CA, USA, 1988, pp. 49-54

8 Wen, C.C., Chen, C.C., Chen, W., and Jiang, J.: 'Magamp post regulation for flyback converter'. PESC, Vancouver, Canada, 2001, pp. $333-338$

\section{Appendix}

For a cubic equation

$$
d^{3}+p \cdot d^{2}+q \cdot d+r=0
$$

One may reduce (54) to the form by substituting for $d$ with the value, $x-p / 3$.

$$
x^{3}+a \cdot x+b=0
$$

where $a=(1 / 3)\left(3 q-p^{2}\right)$ and $b=(1 / 27)\left(2 p^{3}-9 p q+27 r\right)$.

Equation (54) with $a b \neq 0$ can be always solved by transforming it to the trigonometric identity. Let $x=m$ $\cos \theta$, then

$$
\begin{aligned}
x^{3}+a x+b & =m^{3} \cos ^{3} \theta+a m \cos \theta+b \\
& =4 \cos ^{3} \theta-3 \cos \theta-\cos (3 \theta)=0
\end{aligned}
$$

Hence

$$
\frac{4}{m^{3}}=-\frac{3}{a m}=\frac{-\cos (3 \theta)}{b}
$$

from which it follows that:

$$
\begin{gathered}
m=2 \sqrt{-\frac{a}{3}} \\
\cos (3 \theta)=\frac{3 b}{a m}
\end{gathered}
$$

Any solution $\theta_{1}$, which satisfies (59), will also have the solutions

$$
\theta_{1}+\frac{2 \pi}{3} \text { and } \theta_{1}+\frac{4 \pi}{3}
$$

The roots of (55) are

$2 \sqrt{-\frac{a}{3}} \cos \theta_{1}, 2 \sqrt{-\frac{a}{3}} \cos \left(\theta_{1}+\frac{2 \pi}{3}\right), 2 \sqrt{-\frac{a}{3}} \cos \left(\theta_{1}+\frac{4 \pi}{3}\right)$

Hence, the roots of (54) are

$$
\begin{gathered}
2 \sqrt{-\frac{a}{3}} \cos \theta_{1}-\frac{p}{3}, 2 \sqrt{-\frac{a}{3}} \cos \left(\theta_{1}+\frac{2 \pi}{3}\right) \\
-\frac{p}{3}, 2 \sqrt{-\frac{a}{3}} \cos \left(\theta_{1}+\frac{4 \pi}{3}\right)-\frac{p}{3}
\end{gathered}
$$

